\section{Azacitidin bei komorbiden MDS- und AML-Patienten}

Eine retrospektive Analyse zur Wirksamkeit und Verträglichkeit von Azacitidin $\left(\right.$ Vidaza $^{\circledR}$ ) bei MDS-Patienten mit höherem IPSS-Risiko und moderat eingeschränkter bzw. normaler Nierenfunktion ergab, dass auch diese Patienten von der Behandlung profitieren [Kotasianidis IK et al. EHA 2012;Abstr. 1456]. Nach einem Follow-up von 8,6 Monaten ergab sich bei den Patienten mit eingeschränkter Nierenfunktion zwar ein Trend zu einer höheren Abbruchrate, doch vollendeten sie ähnlich viele Therapiezyklen wie die Patienten mit normaler Nierenfunktion (5 vs. 7; p $=0,13$ ). Der Therapieerfolg hinsichtlich Gesamtansprechen $(54,5$ vs. $60,7 \%$; $\mathrm{p}=$ $0,9)$, ereignisfreiem Überleben, definiert als Zeit vom Therapiebeginn bis zur Transformation in eine AML oder Tod (10,9 vs. 9,5 Monate; $\mathrm{p}=0,8)$, und Gesamtüberleben war in beiden Gruppen vergleichbar.

Doris Berger

Nach Informationen von Celgene

\section{Langzeitüberlebensdaten bei CML überzeugend}

Aktuelle 6-Jahres-Follow-up-Daten der Dosisoptimierungsstudie mit Dasatinib $\left(\right.$ Sprycel $\left.^{\oplus}\right)$ zeigen bei erwachsenen Patienten mit einer Philadelphia-Chromosom-positiven chronisch myeloischen Leukämie (CML) eine Gesamtüberlebensrate von $71 \%$ (95\%-Konfidenzintervall [95\%-KI] 63,5-78,5\%). Untersucht wurden 670 Patienten, die gegenüber Imatinib resistent oder intolerant waren. 49,3\% der Patienten, die Dasatinib $100 \mathrm{mg}$ einmal täglich eingenommen hatten, leben nach sechs Jahren noch progressionsfrei (95\%-KI 40,6$50,8 \%$ ) [Rea D et al. Haematologica. 2012;97(Suppl 1):Abstr. 0199]. Die häufigsten hämatologischen Grad-3/4-Nebenwirkungen (kumuliert über sechs Jahre) waren: Neutropenie (36\%), Thrombozytopenie (24\%) und Anämie (13\%). An nicht-hämatologischen Grad-3/4-Toxizitäten wurden beobachtet: Diarrhö (4,3\%), Müdigkeit (4,3\%), Infektionen $(6,1 \%)$ und Pleuraergüsse (5,3\%). Doris Berger.

Nach Informationen von Bristol-Myers Squibb

HER2-positives Mammakarzinom

\title{
Neue Formulierung: Trastuzumab subkutan
}

\section{Die subkutane Behandlung mit Tras- tuzumab bietet den Patientinnen mehr Lebensqualität}

Trastuzumab $\left(\right.$ Herceptin $\left.^{\circledR}\right)$ ist etablierter Standard in der Therapie des HER2-positiven Mammakarzinoms. Sowohl in der adjuvanten als auch in der metastasierten Therapiesituation bietet der humanisierte, monoklonale Antikörper Patientinnen einen signifikanten Überlebensvorteil. Zukünftig steht eine subkutane Formulierung zur Verfügung.

In der zulassungsrelevanten randomisierten, offenen Phase-III-Studie HannaH war die Äquieffizienz der subkutanen Formulierung von Trastuzumab bei Frauen mit HER2-positivem Mammakarzinom im Frühstadium untersucht worden [Jackisch C et al. EBCC 2012: Abstract 1BA]. Neoadjuvant und postoperativ als Monotherapie erhielten die Patientinnen dreiwöchentlich entweder Trastuzumab subkutan in einer fixen
Dosierung von $600 \mathrm{mg}$ oder die intravenöse Standarddosis $(8 \mathrm{mg} / \mathrm{kg}$ initial gefolgt von $6 \mathrm{mg} / \mathrm{kg}$ als Erhaltungsdosis) kombiniert mit einer Chemotherapie.

Die präoperativ gemessene Wirkstoffkonzentration im Blut war unter der fixen Dosierung von Trastuzumab subkutan mindestens genauso hoch wie unter der intravenösen Formulierung. Auch hinsichtlich der Wirksamkeit sind beide Formulierungen vergleichbar: Die pCRRate lag für die subkutane Formulierung zum Operationszeitpunkt bei 45,4\% und bei $40,7 \%$ im Kontrollarm.

Nach Beyhan Ataseven, München, gewinnen die Patientinnen dadurch, dass Trastuzumab künftig auch subkutan verabreicht werden kann. Zumal sie ebenso sicher ist wie die intravenöse Formulierung und auch keine erhöhte Inzidenz kardialer Ereignisse beobachtet wurde.

Doris Berger

Nach Information von Roche

Metastasiertes Nierenzellkarzinom

\section{Ansprechen korreliert mit Gesamtüberleben}

\section{Die Ansprechrate nimmt unter Sunitinib mit der Therapiedauer weiter zu.}

„Die Patienten mit metastasiertem Nierenzellkarzinom (mRCC) möchten leben und bei bestmöglicher Lebensqualität lange von einer Therapie profitieren“, betonte Berit Eberhardt von der Patientenorganisation „Das Lebenshaus e. V.“ auf einem Post-ASCO-Pressegespräch. Der Einsatz von Tyrosinkinase-Inhibitoren (TKI) beim metastasierten Nierenzellkarzinom hat zu einer Verlängerung des Überlebens der Patienten geführt.

Mit einer objektiven Ansprechrate (ORR) von $47 \%$ und einem medianen $\mathrm{Ge}$ samtüberleben (OS) von mehr als zwei Jahren [Motzer RJ et al. J Clin Oncol. 2009; 27(22):3584-90] ist Sunitinib ein Referenzstandard in der mRCC-Erstlinientherapie. Nach einer von Viktor Grünwald, Hannover, auf dem ASCO 2012 vorgestellten Un- tersuchung, korreliert das Ansprechen auf einen TKI in der mRCC-Erstlinientherapie mit dem Gesamtüberleben [J Clin Oncol. 2012;30 (suppl):abstr. 4631]. Aus seiner Sicht ist das Ansprechen daher ein wichtiger Wirksamkeitsparameter - und damit ein relevantes Kriterium für die Therapieentscheidung. Denn letztlich, so Grünwald, gehe es in der modernen mRCC-Therapie um langfristige Wirksamkeit. Dazu sei es wichtig, die Behandlung mit einem effektiven Medikament zu beginnen und sie im Falle eines Ansprechens so lange wie möglich fortzusetzen, zumal die Ansprechrate unter Sunitinib mit der Therapiedauer zunehme. So zeigte sich nach sechs Wochen bei $26 \%$ der Patienten ein Ansprechen, nach 24 Wochen hatten $86 \%$ angesprochen [Molina AM et al. J Clin Oncol. 2012; 30 (suppl:abstr. 4542].

Doris Berger

Nach Informationen von Pfizer Pharma 